\title{
Determination of surface wave velocities in a prestressed anisotropic solid
}

\author{
J.-F. Chai ${ }^{*}$ and T.-T. $\mathrm{Wu}^{\dagger}$ \\ "National Center for Research on Earthquake Engineering, 1 Roosevelt Road, Sec. 4, Taipei, \\ Taiwan \\ ${ }^{\dagger}$ Institute of Applied Mechanics, National Taiwan University, Taipei, Taiwan
}

\begin{abstract}
In this paper, the velocities of surface waves propagating in a prestressed anisotropic crystal are determined both theoretically and experimentally. The Barnett-Lothe's integral formalism, which is fast and efficient in determining the surface wave velocities, is extended to solve the surface wave problem of a prestressed anisotropic material. The governing equations and boundary conditions of the wave superposed on a prestressed elastic body are derived by acousto-elasticity, and the effective wave propagating constants of the finite deformed body are determined. As the effective constants are determined and utilized to replace the elastic constants in the Barnett-Lothe's integral formalism, the surface wave velocities of the prestressed anisotropic body can be determined. In the experiment, the surface wave velocity of a magnesium oxide (MgO) single crystal with (001) orientation under compressive stress is measured. A uniaxial compression in the [100] direction is applied to the crystal, and the corresponding phase velocities of the surface wave propagating on the (001) surface are measured by the $V(z)$ curves of a line focused scanning acoustic microscope (SAM) with a frequency $1.0 \mathrm{GHz}$. Copyright (C) 1996 Elsevier Science Ltd.
\end{abstract}

Keywords: surface wave, acousto-elasticity, scanning acoustic microscope

Ultrasonic surface waves have been applied successfully in many NDE (non-destructive evaluation) problems ${ }^{[1-3]}$. One advantage of using surface waves is that the surface wave energy is confined within a thin surface layer and thus it can propagate a longer distance without attenuation. The other advantage is that both the source and receiver are on the same surface of the specimen and can be applied in some instances where the opposite surface of the specimen is not accessible. The early studies of the propagation of elastic surface waves in crystals can be found in a review article by Farnel1 ${ }^{[2]}$. Basically, most of the studies in the anisotropic surface wave problems adopted the formulation utilized by Synge ${ }^{[4]}$, and hence iterations between the secular equations of the equations of motion and the complex surface boundary determinants are needed to determine the surface wave velocity. The iteration process in the aforementioned formulation is complicated and there is

Corresponding author: Dr Juin-Fu Chai no a priori way to know the existence of surface wave type solution. In a series of papers ${ }^{[5-8]}$, Barnett, Lothe and their coworkers have extended the Stroh formalism ${ }^{[9]}$ to the 'integral formalism' for solving the surface wave problem. In the Stroh formalism, the material properties and the elastic symmetries are contained in the so-called fundamental elasticity tensor, and the solution of a particular problem can be expressed in terms of the eigenvalues and eigenvectors of a six-dimensional tensor of rank two. In the Barnett-Lothe's integral formalism, the surface wave velocity in an anisotropic material can be determined by the vanishing of the determinant of a real symmetric $2 \times 2$ matrix without solving the eigenvalue problem in the Stroh formalism. A review of the integral formalism of surface waves in anisotropic materials and its application to recover the elastic constants and orientations of anisotropic crystals can be found in the papers by $\mathrm{Wu}$ and Chail10,11].

The linear wave equations and constitutive laws are used to express the dynamic motion of an infinitesimal 
deformation, in general. However, as the material is under finite deformation, the relationship of stress and strain becomes complicated and no longer linear. For a nonpiezoelectric material, the nonlinear constitutive laws are derived from the free energy as well as the second law of thermodynamics ${ }^{[12]}$. For an infinitesimal wave superposed on an elastic body under finite deformation, the relationship between the incremental stress and the incremental strain due to the perturbation can be derived by acoustoelasticity ${ }^{[13-15]}$. In the theory of acoustoelasticity, the undeformed reference configuration is called the natural state, the finite deformed state due to the homogeneous prestress is called the initial state, and the current configuration when a dynamic perturbation is superposed on the initial state is called the final state. It is shown that the linearized governing equations of the incremental displacements due to the wave excited in the initial state have the same form as the linear wave equations in the natural state, but the elastic constants in the linear wave equations are replaced by the effective elastic coefficients in the initial state. The effective elastic coefficients are functions of the stress and strain in the initial state as well as the second and the third order elastic constants of the crystal ${ }^{[16,17]}$.

The first study of surface waves excited in a finite deformed body is by Hayes and Rivlin ${ }^{[18]}$, where the propagation of surface waves in an isotropic half-space under homogeneous prestress was studied. Iwashimizu and Kobori ${ }^{[19]}$ determined the variation of surface wave velocities due to the prestress. Chadwick and Jarvis ${ }^{[20]}$ utilized the Barnett-Lothe's integral formalism to study the propagation of surface waves in a prestressed Hadamard isotropic material. The surface wave propagating in a finitely deformed anisotropic material was first studied by Mase and Johnson ${ }^{[21]}$, where the acoustoelasticity and the integral formalism were combined to determine the surface wave velocities.

In recent years, the scanning acoustic microscope $(\mathrm{SAM})^{[22,23]}$ has been developed to observe the surface and subsurface microstructure of solid samples. In addition to the image scanning, SAM has been applied extensively in the analysis of material properties through the development of AMS (acoustic material signature $)^{[24]}$. Basically, AMS is caused by the interference of the leaky surface wave and the reflected wave from the sample surface; the signal intensity thus varies periodically with the vertical position of acoustic lens. This phenomenon is called AMS, and the plot of transducer output voltage versus the lens position is called the $V(z)$ curve $^{[25]}$. From the period of the dips in the $V(z)$ curve, the surface wave velocity of a half-space or a layered sample can be determined through a simple formulation[26], and then the elastic properties of the solid sample can be determined ${ }^{[27]}$.

In this paper, the surface wave excited in a prestressed anisotropic crystal is studied. The governing equations of the incremental displacements due to the wave perturbation are derived, and solved by the Barnett-Lothe's integral formalism to determine the surface wave velocities. The integral formalism and the acoustoelasticity are introduced briefly, firstly, and then the extension of the integral formalism is developed to solve the surface wave problem for a prestressed anisotropic material. A numerical calculation is followed, and the surface wave velocities of silicon ( $\mathrm{Si}$ ) and magnesium oxide ( $\mathrm{MgO})$ crystals are determined. Finally, an $\mathrm{MgO}$ single crystal with (001) orientation is studied in the experiment. Uniaxial compression is applied on the crystal by a screw loading device, and the corresponding phase velocities of the surface wave are measured by the $V(z)$ curves of a line focused scanning acoustic microscope (SAM) with a frequency of $1.0 \mathrm{GHz}$.

\section{Integral formalism}

The conventional way of solving anisotropic surface wave problems was based on the formulation developed by Synge ${ }^{4}$. Iterations between the equations of motion and the complex surface boundary determinants are needed to determine the surface wave velocity. Instead of using the conventional method, the so-called BarnettLothe's integral formalism ${ }^{[5-8]}$ can be utilized for the rapid calculation of the surface wave velocities of an anisotropic material. In the Barnett-Lothe's integral formalism, the surface wave velocity in an anisotropic material can be determined by the vanishing of the determinant of a real symmetric $2 \times 2$ matrix. In the following, only the equations which are needed in the calculation of anisotropic surface waves are given.

For an anisotropic linear elastic material with no body force, the equations of motion are:

$$
C_{i j k l} u_{k, l i}=\rho \ddot{u}_{j}
$$

where $u_{j}$ is the displacement vector, $\rho$ is the mass density, and $C_{i j k l}$ is the anisotropic elastic constant tensor. Consider a surface wave propagating along $\mathbf{m}^{\circ}$ on the surface of an anisotropic half-space with inner normal $n^{\circ}$ (Figure 1), the displacement $u_{i}$ can be expressed as:

$$
u_{i}=U_{i} \exp \left\{\mathrm{i} k\left(m_{l}^{\circ} x_{l}+p n_{l}^{\circ} x_{l}-v t\right)\right\}
$$

where $U_{i}$ is the amplitude of the displacement vector $u_{i}, k$ is the wave number, $v$ is the phase velocity, and $p$ is a complex constant governing the decaying of the amplitude along depth. Substituting Eq. (2) into Eq. (1), yields:

$$
C_{i j k l}^{\prime} u_{k, l i}=0
$$

where $C_{i j k l}^{\prime}$ is the dynamic elastic constant tensor, satisfying:

$$
C_{i j k l}^{\prime}=C_{i j k l}-\rho v^{2} m_{i}^{o} m_{l}^{o} \delta_{j k}
$$

and $\delta_{j k}$ is the Kronecker delta.

Consider the traction vector $\mathbf{T}$ applied on the plane parallel to the surface of the half-space with normal $\mathbf{n}^{\circ}$, it 


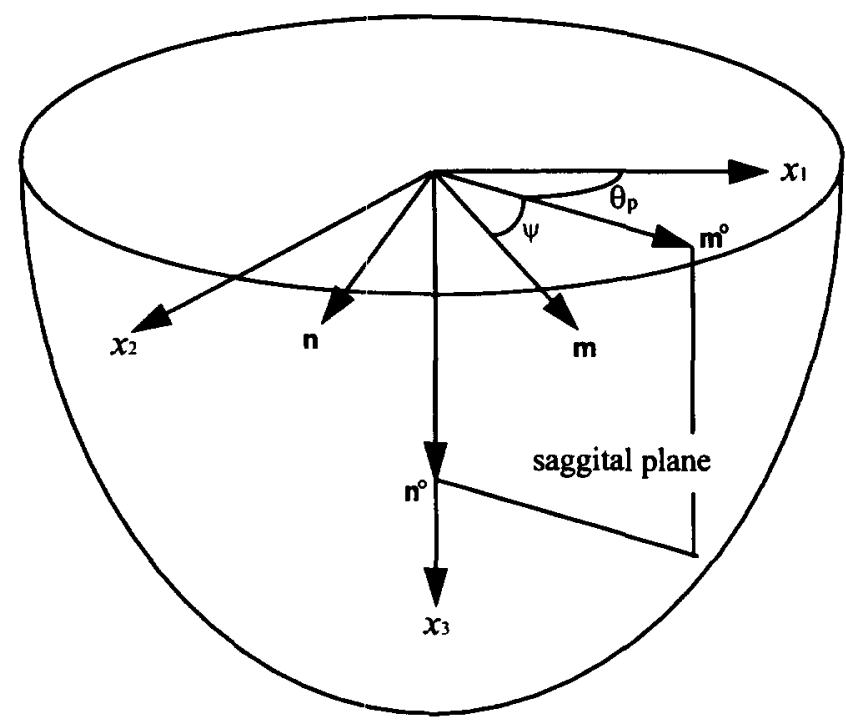

Figure 1 Propagating direction of the surface wave and the coordinate system

can be expressed as:

$$
T_{j}=C_{i j k l} u_{k, l} n_{i}^{o}
$$

Using Eq. (2), and the crthogonal relation $m_{j}^{o} n_{j}^{o}=0$, Eq. (5) can be expressed as:

$$
T_{j}=-\mathrm{i} k V_{j} \exp \left\{\mathrm{i} k\left(m_{l}^{\mathrm{o}} x_{l}+p n_{l}^{\mathrm{o}} x_{l}-v t\right)\right\}
$$

where $V_{j}$ is the amplitude of the traction vector $T_{j}$, satisfying:

$$
V_{j}=-\left[\left(\mathbf{n}^{\mathrm{o}} \mathbf{m}^{\mathrm{o}}\right)+p\left(\mathbf{n}^{\mathrm{o}} \mathbf{n}^{\mathrm{o}}\right)\right]_{j k} U_{k}
$$

and the $3 \times 3$ matrix $(\mathbf{a b})_{j k}$, formed by two vectors $\mathbf{a}$ and b, defined as:

$$
(\mathbf{a b})_{j k}=a_{i} C_{i j k l}^{\prime} b_{l}
$$

By the definition of Eq. (8), motion equations Eq. (3) can be written as:

$$
\left\{p^{2}\left(\mathbf{n}^{\circ} \mathbf{n}^{o}\right)+p\left[\left(\mathbf{m}^{\circ} \mathbf{n}^{o}\right)+\left(\mathbf{n}^{\circ} \mathbf{m}^{\circ}\right)\right]+\left(\mathbf{m}^{\circ} \mathbf{m}^{\circ}\right)\right\}_{j k} U_{k}=0
$$

Introducing a six-dimensional vector $\xi$ :

$$
\xi=\left\{\begin{array}{l}
\mathbf{U} \\
\mathbf{V}
\end{array}\right\}
$$

and combining Eqs. (7) and (9), the so-called sixdimensional eigenvalue problem ${ }^{[9]}$ can be written as:

$$
\mathbf{N}^{\circ} \xi=p \xi
$$

where $\mathbf{N}^{\circ}$ is a $6 \times 6$ matrix:

$$
\begin{aligned}
& \mathbf{N}^{\circ}= \\
& -\left[\begin{array}{cc}
\left(\mathbf{n}^{\circ} \mathbf{n}^{\circ}\right)^{-1}\left(\mathbf{n}^{\circ} \mathbf{m}^{\circ}\right) & \left(\mathbf{n}^{\circ} \mathbf{n}^{\circ}\right)^{-1} \\
\left(\mathbf{m}^{\circ} \mathbf{n}^{0}\right)\left(\mathbf{n}^{0} \mathbf{n}^{\circ}\right)^{-1}\left(\mathbf{n}^{\circ} \mathbf{m}^{\circ}\right)-\left(\mathbf{m}^{\circ} \mathbf{m}^{\circ}\right) & \left(\mathbf{m}^{\circ} \mathbf{n}^{\circ}\right)\left(\mathbf{n}^{\circ} \mathbf{n}^{\circ}\right)^{-1}
\end{array}\right](12)
\end{aligned}
$$

It is noted that the six eigenvalues $p_{\alpha}(\alpha=1 \sim 6)$ of $\mathbf{N}^{\circ}$ occur in three complex conjugate pairs, and so are the corresponding eigenvectors $\xi_{\alpha}$.

Consider the sagittal plane formed by $\mathbf{m}^{\circ}$ and $\mathbf{n}^{\circ}$ (Figure
1), and rotate $\mathbf{m}^{\circ}$ and $\mathbf{n}^{\circ}$ through an angle $\psi$ around the $x_{2}$ axis to a new basis $\mathbf{m}$ and $\mathbf{n}$ in this plane, i.e,

$$
\begin{aligned}
& \mathbf{m}=\mathbf{m}^{\circ} \cos \psi+\mathbf{n}^{\circ} \sin \psi \\
& \mathbf{n}=-\mathbf{m}^{\circ} \sin \psi+\mathbf{n}^{\circ} \cos \psi
\end{aligned}
$$

Now, using the vectors $\mathbf{m}$ and $\mathbf{n}$ to replace the vectors $\mathbf{m}^{\circ}$ and $\mathbf{n}^{\mathbf{0}}$ in Eq. (12), the matrix $\mathbf{N}^{\mathrm{O}}$ becomes a function of the rotation angle $\psi$, and is denoted as $\mathbf{N}$. It can be shown ${ }^{[7]}$ that the eigenvalues of $\mathbf{N}$ are dependent on $\psi$, while the eigenvectors of $\mathbf{N}$ are invariant to $\psi$. Hence, the eigenvalue problem of Eq.(11) becomes:

$$
\mathbf{N}(\psi) \xi_{\alpha}=p_{\alpha}^{\prime}(\psi) \xi_{\alpha}
$$

where $p_{\alpha}^{\prime}$ is the $\alpha$-th eigenvalue of $\mathbf{N}$. The dependence of the eigenvalues $p_{\alpha}^{\prime}$ can be removed and the following form results ${ }^{[7]}$ :

$$
\overline{\mathbf{N}} \xi_{\alpha}= \pm \mathrm{i} \xi_{\alpha}
$$

where:

$$
\overline{\mathbf{N}}=\frac{1}{2 \pi} \int_{0}^{2 \pi} \mathbf{N}(\psi) \mathrm{d} \psi
$$

The choice of \pm is dependent on whether $p_{\alpha}^{\prime}$ has a positive or negative imaginary part. The components of $\overline{\mathbf{N}}$ are denoted as:

$$
\overline{\mathbf{N}}=\left[\begin{array}{ll}
\mathbf{S} & \mathbf{Q} \\
\mathbf{B} & \mathbf{S}^{\mathrm{T}}
\end{array}\right]
$$

and:

$$
\begin{aligned}
& \mathbf{S}=-\frac{1}{2 \pi} \int_{0}^{2 \pi}(\mathbf{n n})^{-1}(\mathbf{n m}) \mathrm{d} \psi \\
& \mathbf{Q}=-\frac{1}{2 \pi} \int_{0}^{2 \pi}(\mathbf{n n})^{-1} \mathrm{~d} \psi \\
& \mathbf{B}=-\frac{1}{2 \pi} \int_{0}^{2 \pi}\left[(\mathbf{m n})(\mathbf{n n})^{-1}(\mathbf{n m})-(\mathbf{m m})\right] \mathbf{d} \psi
\end{aligned}
$$

The limiting velocity $v_{\mathrm{L}}$ is defined as the smallest velocity for which (nn) $)^{-1}$ becomes singular for some orientation of $\mathbf{n}$ in the sagittal plane (with angle $\psi_{\mathrm{L}}$ ), thus in the subsonic range $\left(0 \leq v<v_{\mathrm{L}}\right),(\mathbf{n n})^{-1}$ and therefore, $\mathbf{N}$ and $\overline{\mathbf{N}}$ always exist. From the definition of (nn), one can show that $v_{\mathrm{L}}=c_{1}\left(\psi_{\mathrm{L}}\right) \sec \left(\psi_{\mathrm{L}}\right)$, where $c_{1}\left(\psi_{\mathrm{L}}\right)$ is the smallest bulk wave velocity propagating along the $\psi_{\mathrm{L}}$ direction.

The boundary condition on the surface of the half-space is traction free, i.e.:

$$
T_{j}=C_{i j k l} u_{k, l} n_{i}^{\mathrm{o}}=0 \quad \text { on } x_{3}=0
$$

It has been shown ${ }^{[7]}$ that matrix $\mathbf{B}$ is real symmetric and its rank becomes one when the traction free boundary condition, Eq. (19), is satisfied. Thus, the determinants of all the $2 \times 2$ submatrix of $\mathbf{B}$ are zero, for example:

$$
\left|B_{11} B_{22}-B_{12} B_{21}\right|=0
$$

It follows that the finding of the surface wave velocity of an anisotropic solid can be achieved simply by finding of velocity which causes the determinant of a $2 \times 2$ real matrix vanishing. 
Once the surface wave velocity along a particular propagation direction $\mathbf{m}^{\circ}$ is known, the distributions of the displacement and traction along the depth of an anisotropic half-space are determined straightforwardly by solving the eigenvalue problem as shown in Eq. (11). For a surface wave propagating in a half-space with $n_{j}^{o} x_{j} \geq 0$, by Eq. (2), only those eigenvalues $p_{\alpha}$ $(a=1 \sim 3)$ with positive imaginary parts are retained in the construction of the surface wave. Hence, the displacement and traction can be expressed as:

$$
\begin{aligned}
& u_{i}=\sum_{\alpha=1}^{3} C_{\alpha} U_{i \alpha} \exp \left\{\mathrm{i} k\left(m_{l}^{\mathrm{o}} x_{l}+p_{\alpha} n_{l}^{\mathrm{o}} x_{l}-v t\right)\right\} \\
& T_{i}=-\mathrm{i} k \sum_{\alpha=1}^{3} C_{\alpha} V_{i \alpha} \exp \left\{\mathrm{i} k\left(m_{l}^{\mathbf{o}} x_{l}+p_{\alpha} n_{l}^{\mathrm{o}} x_{l}-v t\right)\right\}
\end{aligned}
$$

where $U_{i \alpha}$ and $V_{i \alpha}$ are the $\alpha$-th $(\alpha=1 \sim 3)$ complex eigenvectors associated with the eigenvalues $p_{\alpha}$, and $C_{\alpha}$ are constants to be determined from the traction free boundary conditions, written as:

$$
\sum_{\alpha=1}^{3} C_{\alpha} V_{i \alpha}=0
$$

\section{Acoustoelasticity}

In order to study the propagation of surface waves in a prestressed anisotropic crystal, the governing equations and boundary conditions for the surface wave are required. Compared with the predeformation, the surface wave can be recognized as a perturbation, therefore the acoustoelasticity is applied to derive the governing equations for the small-field variables of the surface wave which is superposed on the prestressed elastic body. In fact, the governing equations derived in this section are generally applied for all small dynamic fields superposed on a predeformed anisotropic material, and the surface wave perturbation is one of the important special cases.

To distinguish the deformation clearly, three deformation states are defined as follows. The undeformed reference configuration is called the natural state, and the finite deformation due to the homogeneous prestress will transform the configuration to the initial state, and the homogeneous initial state is in a static equilibrium. At the current time, a dynamic perturbation is superposed on the initial state, and then the configuration is changed from the initial state to the final state. As shown in Figure 2, a common Cartesian coordinate system is used and the position vectors of the material point in the natural, initial and final states are denoted as $\xi, \mathbf{X}$, and $\mathbf{x}$, respectively. The components of physical quantities which refer to the natural state are denoted by Greek subscripts, and those which refer to the initial and final states by capital and lower case Roman subscripts, respectively. For example, $\xi_{\alpha}, X_{K}$ and $x_{k}$ $(\alpha, K, k=1,2,3)$ are the components of position vectors

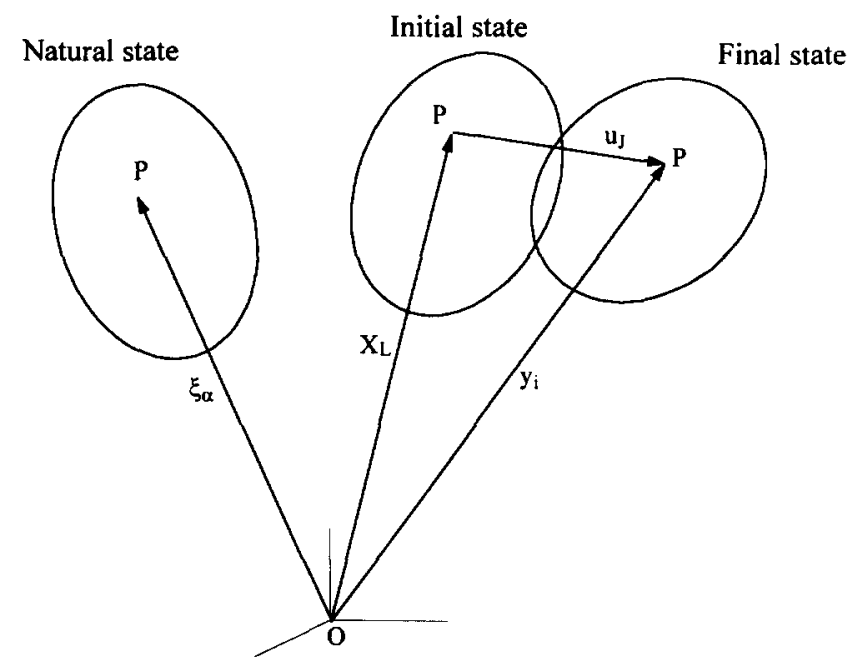

Figure 2 Natural state, initial state, and final state

in the respective configurations. The superscripts $i$ and $f$ of physical quantities denote that the functions are evaluated in the initial state and the final state, respectively.

The deformation of the body can be described as a oneto-one mapping:

$$
\begin{aligned}
X_{\mathrm{L}} & =X_{\mathrm{L}}\left(\xi_{\alpha}\right) \\
x_{i} & =x_{i}\left(X_{\mathrm{L}}, t\right)
\end{aligned}
$$

and then the infinitesimal displacement from the initial state to the final state is written as:

$$
u_{I}\left(X_{\mathrm{L}}, t\right)=\delta_{i I} x_{i}\left(X_{\mathrm{L}}, t\right)-X_{I}
$$

The initial state is in a static equilibrium, the equations of motion are:

$$
\tau_{I J, I}^{\mathrm{i}}=0
$$

and the final state is in a dynamic equilibrium, the equations of motion are:

$$
\tau_{i j, i}^{\mathrm{f}}=\rho^{\mathrm{f}} \ddot{u}_{j}
$$

where $\tau_{I J}^{\mathrm{i}}$ and $\tau_{i j}^{\mathrm{f}}$ are the Cauchy stress tensors in the initial state and final state, respectively, and $\rho^{\mathrm{f}}$ is the mass density in the final state.

In the general case, the deformation from the natural state to the initial state has been determined, and therefore the position vector and material boundary in the initial state are well defined. It is noted that Eq. (26) is referred to the final configuration, however the deformation and position vector in the final state are still unknown before the boundary value problem is determined. In order to specify the boundary condition and solve the problem more easily, it is convenient to derive the equations of motion referred to the initial configuration. The Cauchy stress tensor in the final state can be transformed to refer to the initial configuration and become the second kind of Piola-Kirchhoff stress tensor, denoted as $S_{I J}^{f}$. The relationship between $\tau_{i j}^{f}$ and 
$S_{I J}^{f}$ is:

$$
\tau_{i j}^{\mathrm{f}}=\frac{1}{J} x_{i, I} x_{j, J} S_{I J}^{\mathrm{f}}
$$

and therefore, the equations of motion in the final state (Eq. (26)) can be derived to refer to the initial configuration; it reads:

$$
\frac{\partial}{\partial X_{I}}\left(S_{I J}^{\mathrm{f}}+\frac{\partial u_{J}}{\partial X_{L}} S_{I L}^{\mathrm{f}}\right)=\rho^{i} \ddot{u}_{J}
$$

where $\rho^{\mathrm{i}}$ is the mass derisity in the initial state, satisfying:

$$
\rho^{\mathrm{i}}=J \rho^{\mathrm{f}}
$$

and $J$ is the Jacobian, defined as the determinant of the deformation gradient $x_{i, I}$.

Define the increment of stress tensor $S_{I J}$ as:

$$
S_{I J}^{\mathrm{f}}=\tau_{I J}^{\mathrm{i}}+S_{I J}
$$

then, by Eqs. (25) and (30), Eq. (28) becomes

$$
\frac{\partial}{\partial X_{I}}\left(S_{I J}+\frac{\partial u_{J}}{\partial X_{\mathrm{L}}} \tau_{I L}^{\mathrm{i}}\right)=\rho^{i} \ddot{u}_{J}
$$

where the product of small variables $S_{I L} u_{J, L}$ has been neglected.

Furthermore, the constitutive equations between the incremental stress tensor $S_{I J}$ and the displacement $u_{I}$ are derived. To satisfy the axiom of the objective, all the physical quantities are referred to the undeformed natural configuration. The Cauchy stress tensors in the initial and final states, $\tau_{I J}^{\mathrm{i}}$ and $\tau_{i j}^{\mathrm{f}}$, are both transformed to refer to the natural configuration as the second kind of Piola-Kirchoff stress tensors, denoted as $S_{\alpha \beta}^{\mathrm{i}}$ and $S_{\alpha \beta}^{\mathrm{f}}$, respectively. The constitutive equations can be derived from the stored energy function as ${ }^{[12]}$ :

$$
\begin{aligned}
S_{\alpha \beta}^{\mathrm{i}} & =C_{\alpha \beta \gamma \delta} E_{\gamma \delta}^{\mathrm{i}}+\frac{1}{2} C_{\alpha \beta \gamma \delta \epsilon \eta} E_{\gamma \delta}^{\mathrm{i}} E_{\epsilon \eta}^{\mathrm{i}} \\
S_{\alpha \beta}^{\mathrm{f}} & =C_{\alpha \beta \gamma \delta} E_{\gamma \delta}^{\mathrm{f}}+\frac{1}{2} C_{\alpha \beta \gamma \delta \epsilon \eta} E_{\gamma \delta}^{\mathrm{f}} E_{\epsilon \eta}^{\mathrm{f}}
\end{aligned}
$$

where $C_{\alpha \beta \eta \delta}$ and $C_{\alpha \beta \gamma \delta \varepsilon \eta}$ are the second and third order elastic constants, respectively, and $E_{\gamma \delta}^{\mathrm{i}}$ and $E_{\gamma \delta}^{\mathrm{f}}$ are the Lagrange finite strain tensors in the initial and final states, respectively.

Therefore, the interest incremental Piola-Kirchoff stress tensor $S_{I J}$ can be obtained by:

$$
S_{I J}=\frac{1}{J^{\mathrm{i}}} X_{I, \alpha} X_{J, \beta} S_{\alpha \beta}
$$

where $S_{\alpha \beta}$ is the increment of the stress from the initial state to the final state, i.e.:

$$
S_{\alpha \beta}=S_{\alpha \beta}^{\mathrm{f}}-S_{\alpha \beta}^{\mathrm{i}}
$$

and $J^{\mathrm{i}}$ is the Jacobian from the natural state to the initial state. The inverse of $J^{i}$ can be approximated to the linear terms as:

$$
\frac{1}{J^{\mathrm{i}}}=1-u_{K, K}^{\mathrm{i}}
$$

Substituting Eqs. (34), (35) and (32) into Eq. (33), and using the chain rule and neglecting the higher order terms, we obtain:

$$
S_{I J}=\bar{C}_{I J K L} u_{K, L}
$$

with:

$$
\begin{aligned}
\bar{C}_{I J K L}= & C_{I J K L}\left(1-u_{N, N}^{\mathrm{i}}\right)+C_{I J K M} u_{L, M}^{\mathrm{i}} \\
& +C_{I J M L} u_{K, M}^{\mathrm{i}}+C_{I M K L} u_{J, M}^{\mathrm{i}} \\
& +C_{M J K L} u_{I, M}^{\mathrm{i}}+C_{I J K L M N} u_{M, N}^{\dot{\mathrm{i}}_{M}}
\end{aligned}
$$

and:

$$
\begin{aligned}
& C_{I J K L}=\delta_{I \alpha} \delta_{J \beta} \delta_{K \gamma} \delta_{L \delta} C_{\alpha \beta \gamma \delta} \\
& C_{I J K L M N}=\delta_{I \alpha} \delta_{J \beta} \delta_{K \gamma} \delta_{L \delta} \delta_{M \epsilon} \delta_{N \eta} C_{\alpha \beta \gamma \delta \epsilon \eta}
\end{aligned}
$$

Combining the equations of motion (Eq. (31)) and the constitutive equations (Eq. 36)), we have:

$$
\frac{\partial}{\partial X_{I}}\left\{\left(\bar{C}_{I J K L}+\tau_{I L}^{\mathrm{i}} \delta_{J K}\right) u_{K, L}\right\}=\rho^{\mathrm{i}} \ddot{u}_{J}
$$

Since the initial state is homogeneous, the initial stress $\tau_{I L}^{\mathrm{i}}$ and the initial displacement gradient $u_{L, M}^{\mathrm{i}}$ are constant and independent of the spatial coordinate, Eq. (39) yields:

$$
A_{I J K L} u_{K, L I}=\rho \mathrm{i} \ddot{u}_{J}
$$

where $A_{I J K L}$ is recognized as the effective material constants in the prestressed initial state, expressed as:

$$
A_{I J K L}=\bar{C}_{I J K L}+\tau_{I L}^{\mathrm{i}} \delta_{J K}
$$

The surface of the half-space is traction free both in the initial state and the final state. The surface traction in the initial state is

$$
T_{J}^{\mathrm{i}}=\tau_{I J}^{\mathrm{i}} N_{I} \quad \text { on } X_{3}=0
$$

and in the final state is:

$$
T_{j}^{\mathrm{f}}=\tau_{i j}^{\mathrm{f}} n_{i} \quad \text { on the wave surface }
$$

where $N_{I}$ and $n_{i}$ are the unit normal of the half-space surface in the initial and final states, respectively. Furthermore, the surface traction in the final state can be expressed referred to the initial configuration as ${ }^{[15]}$ :

$$
T_{J}^{\mathrm{f}}=S_{I J}^{\mathrm{f}} N_{I} \quad \text { on } X_{3}=0
$$

Since the boundary conditions in both the initial state and the final state are traction free, we have:

$$
\begin{array}{ll}
\tau_{I J}^{\mathrm{i}} N_{I} & =0 \\
S_{I J}^{\mathrm{f}} N_{I} & =0
\end{array} \quad \text { on } X_{3}=0
$$

By Eqs. (30), (36), (45), the boundary condition for the incremental field due to the wave perturbation can be written as:

$$
\left(\bar{C}_{I J K L}+\tau_{I L}^{\mathrm{i}} \delta_{J K}\right) u_{K, L} N_{I}=0 \quad \text { on } X_{3}=0
$$

and from Eq.(41), Eq. (46) becomes

$$
A_{I J K L} u_{K, L} N_{I}=0 \quad \text { on } X_{3}=0
$$


Consider the special case of no predeformation, the initial state is identical with the natural state, and then the stress tensor and initial displacement gradient in the initial state are vanishing. Thus, from Eqs. (37) and (41), we obtain:

$$
A_{I J K L}=C_{I J K L}
$$

The mass density $\rho^{\mathrm{i}}$ in the initial state is the same as in the natural state, denoted as $\rho^{\circ}$. Therefore, the differential equations for small displacement $u_{I}$ are reduced to the linear wave equations, and reads:

$$
C_{I J K L} u_{K, L I}=\rho^{\circ} \ddot{u}_{J}
$$

and it is identical with the linear wave equation.

\section{Surface wave in a prestressed anisotropic material}

\section{Extension of Barnett-Lothe's integral formalism}

In this section, the Barnett-Lothe's integral formalism is extended to solve the surface wave problem for a prestressed anisotropic crystal. The governing equations and the boundary conditions for surface waves superposed on a prestressed anisotropic material have been given in Eqs. (40) and (47). Compared with Eqs. (1) and (19), it is observed that the boundary value problem for surface waves excited in the undeformed natural state and in the prestressed initial state have the same form. The only difference is that the elastic constants $C_{i j k l}$ in the natural state are replaced by the effective material constants $A_{I J K L}$ in the initial state. It is suggested that, if the position vector $X_{K}$ is selected to be the spatial coordinates, the integral formalism can be applied to determine the surface wave velocities of the prestressed anisotropic crystal.

The existence and uniqueness of the velocity of surface waves excited in the undeformed elastic body can be proved mathematically in the Barnett-Lothe's integral formalism ${ }^{[7,8]}$. Even for piezoelectric crystals, the surface wave problem can be solved by the integral formalism ${ }^{[28]}$, and a systematic algorithm has been developed to show some properties of the integral matrices, which is used to prove the existence and uniqueness of the surface wave velocities for non-stressed piezoelectric crystals ${ }^{[28]}$. As the material constants (e.g. elastic, piezoelectric and dielectric constants) in the natural state are replaced by the effective material constants in the prestressed initial state, the properties of the integral matrices have been proved $^{[29]}$ to be the same as those for the non-stressed crystals, and then, the existence and uniqueness of surface waves shown by the integral formalism for non-stressed piezoelectric crystals are still applied to the prestressed ones. Therefore, the integral formalism can be applied to solve the surface wave problem for a prestressed piezoelectric crystal ${ }^{[29]}$.
When reduced to non-piezoelectric elastic materials, considered in this paper, the procedures to prove the identity of the properties of integral matrices in the nonstressed and prestressed states are similar to those derived for piezoelectric materials, except that the physical quantities about the electric field are dropped in the procedures. In other words, the surface wave problem for a prestressed anisotropic crystal can be solved without any doubt by the integral formalism.

Since the effective material constants $A_{I J K L}$ are functions of the initial stress $\tau_{I J}^{\mathrm{i}}$, and the initial displacement gradient $u_{I, J}^{\mathrm{i}}$ as shown in Eqs. (37) and (41), the initial displacement gradient must be determined from the specified initial stress firstly. It can be done by using the constitutive equations (32) as well as the relationship:

$$
\tau_{I J}^{\mathrm{i}}=\frac{1}{J^{\mathrm{i}}} X_{I, \alpha} X_{J, \beta} S_{\alpha \beta}^{\mathrm{i}}
$$

and the result form follows:

$$
\begin{aligned}
\tau_{I J}^{\mathrm{i}}= & {\left[C_{I J K L}\left(1-u_{N, N}^{\mathrm{i}}\right)+C_{I J K M} u_{L, M}^{\mathrm{i}}+C_{I M K L} u_{J, M}^{\mathrm{i}}\right.} \\
& +C_{M J K L} u_{I, M}^{\mathrm{i}}+\frac{1}{2} C_{I J M L} u_{K, M}^{\mathrm{i}} \\
& \left.+\frac{1}{2} C_{I J K L M N} u_{M, N}^{\mathrm{i}}\right] u_{K, L}^{\mathrm{i}}
\end{aligned}
$$

If one retains up to linear terms only, Eq. (51) reduces to:

$$
\tau_{I J}^{\mathrm{i}}=C_{I J K L} u_{K, L}^{\mathrm{i}}
$$

As the initial displacement gradient is determined from Eq. (51) or (52), the constants $\bar{C}_{I J K L}$ and then the effective material constants $A_{I J K L}$ can be determined straightforwardly by Eqs. (37) and (41), respectively.

The relationship between the mass densities $\rho^{\mathrm{i}}$ and $\rho^{\circ}$ is:

$$
\rho^{\mathrm{i}}=\frac{1}{J^{\mathrm{i}}} \rho^{\mathrm{o}}=\left(1-u_{M, M}^{\mathrm{i}}\right) \rho^{\mathrm{o}}
$$

therefore, the initial mass density can be determined as the initial displacement gradient is determined.

By the associated effective material constants $A_{I J K L}$ and the initial mass density $\rho^{\mathrm{i}}$ for an anisotropic crystal under specified prestress, the corresponding integral matrix $\mathbf{B}$ in the Barnett-Lothe's integral formalism can be calculated, and then the surface wave velocity is determined by Eq. (20). As mentioned before, the calculated velocity is the unique solution for this problem. The procedures to determine the surface wave velocities of a prestressed crystal are shown in Figure 3.

\section{Numerical implementation}

In this section, the surface wave velocities of single crystals $\mathrm{Si}$ and $\mathrm{MgO}$ with the (001) orientation are calculated. From the symmetry of cubic crystals, the numbers of the independent second and third order elastic constants are three and six, respectively. Most of the components of the elastic constant tensor are 


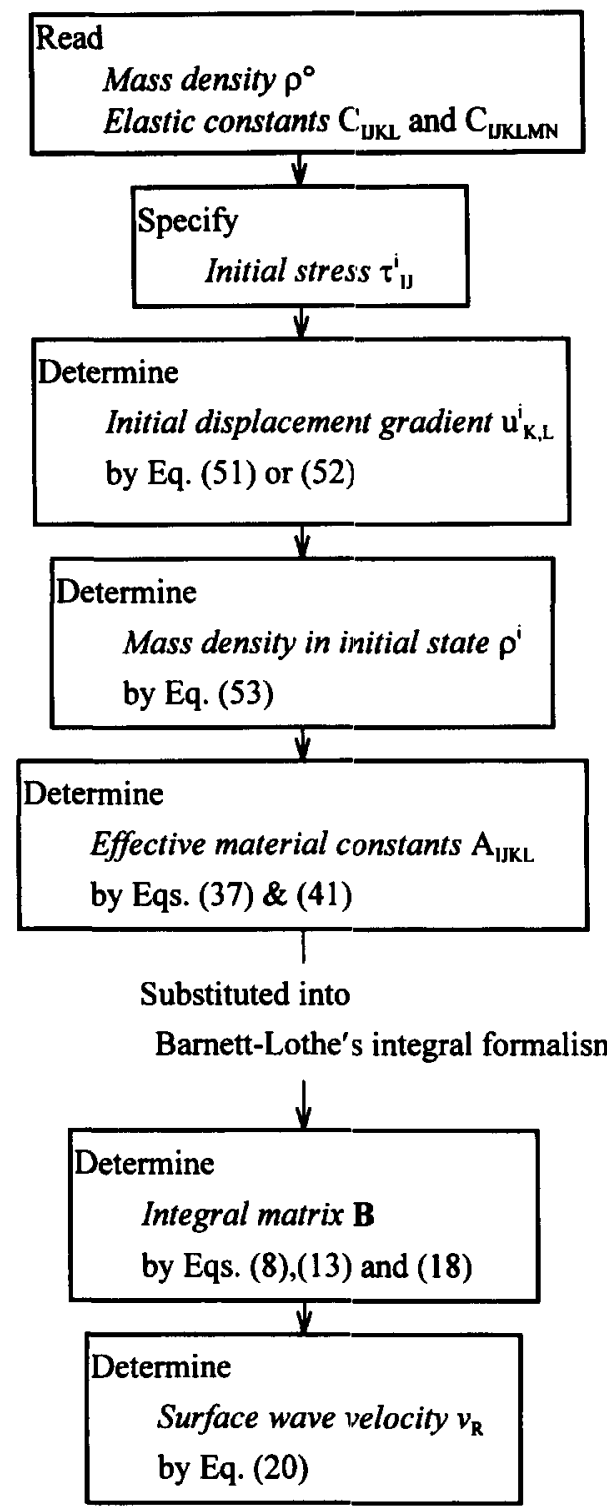

Figure 3 The calculating procedures of the extended BarnettLothe's integral formalism

zero, and the nonzero components are written in the Voigt form as:

$$
\begin{aligned}
& C_{11}=C_{22}=C_{33} \\
& C_{12}=C_{13}=C_{23} \\
& C_{44}=C_{55}=C_{66}
\end{aligned}
$$

and:

$$
\begin{aligned}
& C_{111}=C_{222}=C_{333} \\
& C_{144}=C_{255}=C_{366} \\
& C_{112}=C_{223}=C_{133}=C_{113}=C_{122}=C_{233} \\
& C_{155}=C_{244}=C_{344}=C_{166}=C_{266}=C_{355} \\
& C_{123} \\
& C_{456}
\end{aligned}
$$

The values of the density and these elastic constants for $\mathrm{Si}$ and $\mathrm{MgO}$ are shown in the appendix.

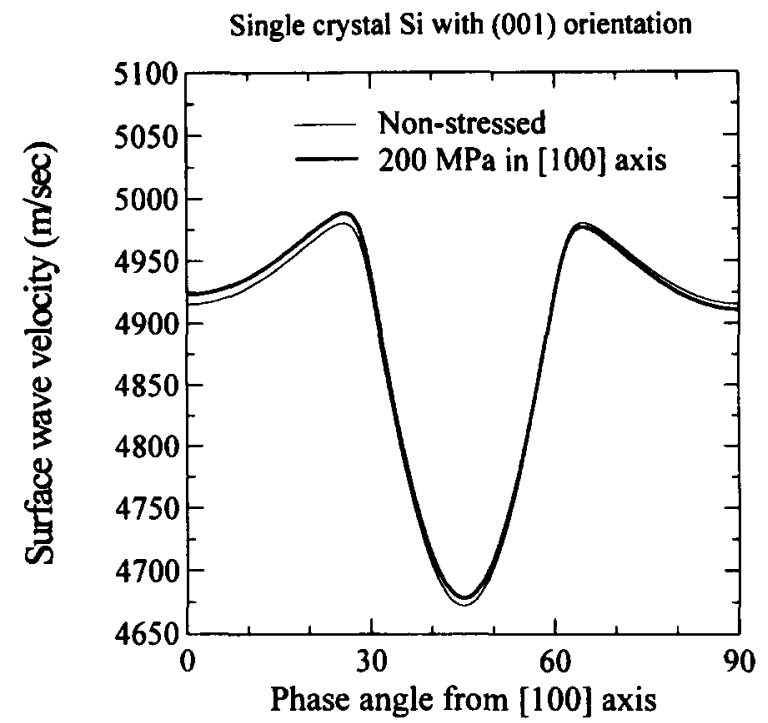

Figure 4 Surface wave velocity of single crystal Si under uniaxial tension in the [100] axis

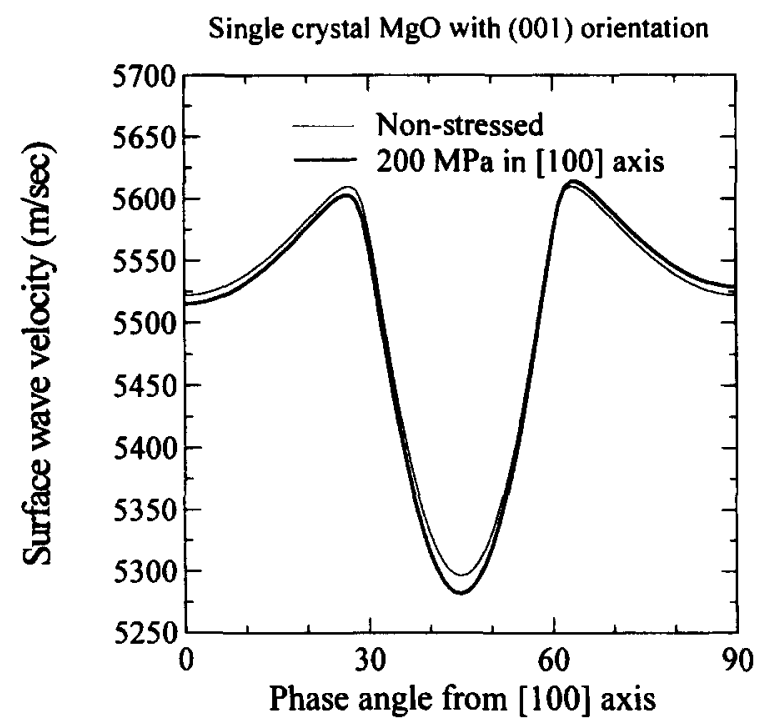

Figure 5 Surface wave velocity of single crystal $\mathrm{MgO}$ under uniaxial tension in the $[100]$ axis

Considering the case of the nonstressed natural state firstly, the density $\rho$ and the second order elastic constants $C_{i j k l}$ are used directly to determine the integral matrix $B$ in the Barnett-Lothe's integral formalism, and the surface wave velocities are determined by Eq. (20). The calculated results for $\mathrm{Si}$ and $\mathrm{MgO}$ with no prestress are shown as the thinner line in Figures 4 and 5, respectively. In Figures 4 and 5, the horizontal axis is the phase angle of the surface wave, ranged from the [100] direction through the [110] to the [010] direction.

In the following, the case of a uniaxial tension stress with magnitude of $200.0 \mathrm{MPa}$ along the [100] direction is considered. Following the flow chart shown in Figure 3, the corresponding effective material constants $A_{I J K L}$, initial mass density $\rho^{\mathrm{i}}$ and the surface wave velocities are determined by the extended Barnett-Lothe's integral formalism under the specified prestress.

The calculated results for $\mathrm{Si}$ and $\mathrm{MgO}$ with uniaxial 
stress $200.0 \mathrm{MPa}$ applied in the [100] direction are shown as the thicker line in Figures 4 and 5, respectively. It is seen from Figure 4 that the velocities for $\mathrm{Si}$ are increased as the surface waves propagating near the [100] axis, parallel to the tension direction, and decreased near the [010] axis, perpendicular to the tension direction. However, the trend of velocity variation due to the same prestress is opposite to the case of $\mathrm{MgO}$ crystal, i.e., the velocity is decreased near the [100] axis and is increased near the [010] axis.

\section{Measurement of surface wave velocities}

\section{$V(z)$ curve and surface wave velocity}

In this paper, the surface wave velocities of a single crystal are measured by a line focused scanning acoustic microscope (SAM). Because of the interference of the leaky surface wave and the directly reflected wave from the surface of a solid sample, the intensity of received signals varies periodically as the lens moves toward the specimen. The plot of the output voltage of the transducer versus the vertical position of the acoustic lens is called the $V(z)$ curve, which is one of the material characteristics (called the acoustic material signature), and the surface wave velocity of the solid sample can be determined from the period of the dips in the $V(z)$ curve.

Shown in Figure 6 is a simple model for the periodic oscillations in the $V(z)$ curve. As the acoustic field is refracted through the lens/couplant boundary and focused onto the solid sample, a surface wave is excited on the sample surface if the following conditions are satisfied simultaneously: (1) the critical angle $\theta_{\mathrm{R}}$ is less than the opening angle $\theta_{\max }$, and (2) the distance between the lens and the sample surface is less than the focal length of the lens. According to ray theory, two major rays are reflected back into the lens and detected by the piezoelectric transducer. One ray, denoted as \#0 in Figure 6, is due to the wave incident near the $z$ axis and reflected directly from the sample surface, the other ray,

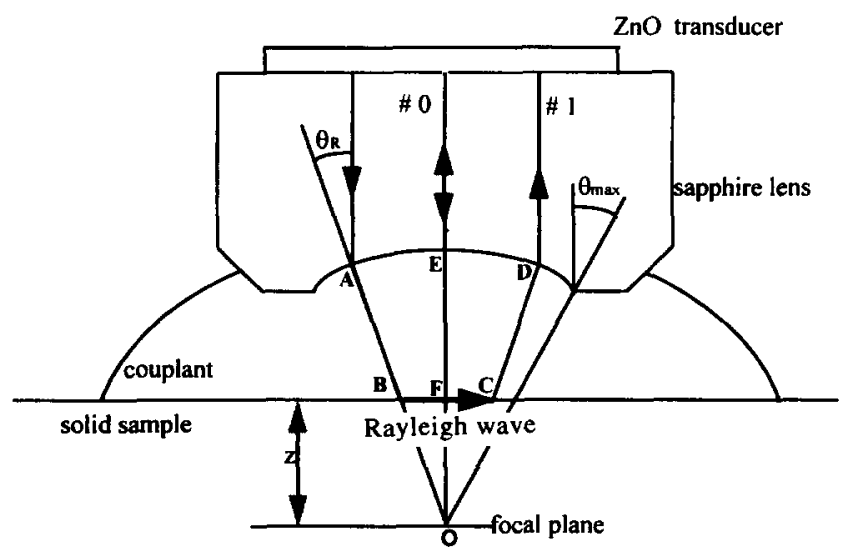

Figure 6 Surface wave and the $V(z)$ curves of scanning acoustic microscope denoted as \#1 in Figure 6, is associated with the leaky surface wave generated by the wave incident at the critical angle. These two rays interfere at the transducer and result in the periodic oscillation in the $V(z)$ curve. The period $\Delta z$ of the dips in the $V(z)$ curve is expressed as ${ }^{[26]}$ :

$$
\Delta z=\frac{v_{\mathrm{c}}}{2 f\left(1-\cos \theta_{\mathrm{R}}\right)}
$$

where $v_{\mathrm{c}}$ is the acoustic wave velocity of couplant, and $f$ is the frequency of the acoustic lens. Since the critical angle is defined as:

$$
\theta_{\mathrm{R}}=\sin ^{-1}\left(\frac{v_{\mathrm{c}}}{v_{\mathrm{R}}}\right)
$$

the Rayleigh wave velocity $v_{R}$ of the solid sample is obtained as:

$$
v_{\mathrm{R}}=v_{\mathrm{c}}\left\{1-\left(1-\frac{v_{\mathrm{c}}}{2 f \Delta z}\right)^{2}\right\}^{-\frac{1}{2}}
$$

In general, the period of the $V(z)$ curve is determined through the utilization of FFT analysis, the centre frequency of the peak in the spectrum is just equal to the inverse of period $\Delta z$. However, the resolution in the frequency domain is limited by the total length of the $V(z)$ curve, and the frequency of a periodic oscillation determined by FFT method is shifted if the number of cycles within the input data is not an integer. In the present study, the total length of the available $V(z)$ curve is limited by the working distance to be less than $60 \mu \mathrm{m}$, and it is difficult to gate integral cycles exactly from the measured $V(z)$ curve, therefore, the $\Delta z$ determined by FFT method is not accurate enough.

Instead of using the conventional FFT analysis, the frequency function formatted by autoregressive model (AR model) is used to obtain the spectrum of the measured $V(z)$ curve, and it is solved by the programs in the System Identification Toolbox of MATLAB software[30]. In this method, the resolution in the spatial frequency domain is controllable by users, independent of the total length of the $V(z)$ curve. Furthermore, the frequency of a period variation determined by AR model is still accurate even if nonintegral cycles are used.

It is noted that the surface wave generated by a line focused acoustic lens is propagating along a specified direction, and then the velocity determined by Eq. (58) is the phase velocity along the specified direction. Therefore, this method is applied to measure the velocities of surface waves propagating along different directions in an anisotropic crystal.

\section{Experimental set-up}

A Leitz 'ELSAM' reflection type scanning acoustic microscope was utilized in this study. A line focused acoustic lens with operating frequency $1.0 \mathrm{GHz}$ was used to measure the period $\Delta z$ in $V(z)$ curves. In the $V(z)$ curve measurement, the specimen is fixed on a horizontal 
stage, and the lens is moved toward the sample vertically from a proper initial position. The working distance is fixed as $60 \mu \mathrm{m}$ and equally separated by 512 steps. In order to create the surface waves on the solid sample, the useful $V(z)$ curve is limited in the so called 'negative defocus' range, where the distance between sample surface and lens is less than the focal length of the acoustic lens. The horizontal stage can be rotated about $30^{\circ}$ around the lens by a mechanical system, therefore, the propagating direction of the surface wave excited by the line focused acoustic lens is well defined. In the ELSAM acoustic microscope, the temperature of the couplant can be heated and maintained at $35^{\circ} \mathrm{C}$ or $60^{\circ} \mathrm{C}$ by a small coil-heater around the lens. The heating device was originally designed to increase the couplant's temperature and hence to improve the signal to noise ratio at high operating frequencies.

The single crystal $\mathrm{MgO}$ (cubic crystal) is used as the specimen in the experiment. The crystal is a rectangular parallelepiped and the unit normal of the six boundaries are parallel to the [100], [010], and [001] axes. The length, width and thickness of the crystal are $20 \mathrm{~mm}, 10 \mathrm{~mm}$, and $10 \mathrm{~mm}$, respectively. Since the frequency of the surface wave generated by the acoustic lens is up to $1.0 \mathrm{GHz}$, the wavelength (about $5.5 \mu \mathrm{m}$ ) is much less than the thickness $(10 \mathrm{~mm})$ in the [001] axis, the crystal can be recognized as a half-sprace with $(001)$ orientation. The detailed geometry and size of the $\mathrm{MgO}$ crystal are shown in Figure 7.

The $\mathrm{MgO}$ crystal is loaded by a uniaxial compression device in order to measure the associated surface wave velocities at the prestressed state. The loading system is shown in Figure 8, where the uniaxial compression is created by an M10 hexagon-head cap screw made by low carbon steel and an aluminium substrate with screw thread. The aluminium block with an electrical resistance strain gauge mounted on the surface is used to measure the uniaxial stress applied on the MgO crystal, and the geometry of the aluminium block is also a rectangular parallelepiped of size $10 \times 10 \times 30 \mathrm{~mm}^{3}$. The alignment of the aluminium block with the $\mathrm{MgO}$ crystal is shown in Figure 8 . We note that with this arrangement, the stresses in the aluminium and $\mathrm{MgO}$ specimens are the same. The uniaxial stress of the $\mathrm{MgO}$ crystal under the screw

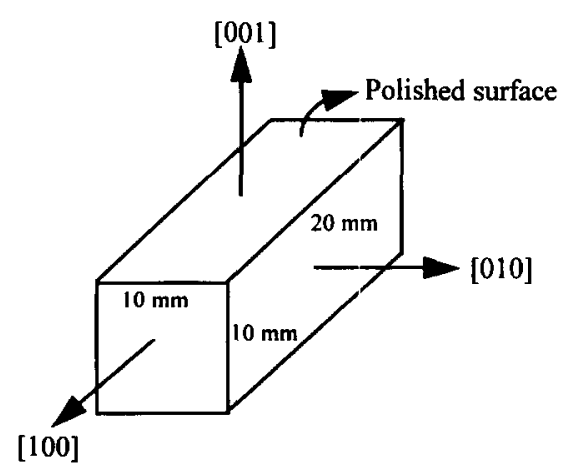

Figure 7 Geometry and size of the MgO crystal in the experiment loading system can then be determined from the product of the Young's modulus $E_{\mathrm{Al}}$ of the aluminium block multiplied by the uniaxial strain $\epsilon_{11}$ measured by the strain gauge as:

$$
\tau_{I J}^{\mathrm{i}}= \begin{cases}E_{\mathrm{Al}} \times \epsilon_{11} & \text { for } I=J=1 \\ 0.0 & \text { otherwise }\end{cases}
$$

The aluminium block is a polycrystal isotropic material, its Young's modulus can be measured by the ultrasonic method. The longitudinal and shear wave velocities of the aluminium block are measured and the Young's modulus of the aluminium block was determined as $E_{\mathrm{Al}}=71.81 \mathrm{GPa}$.

\section{Experimental results}

In order to measure the surface wave velocities of the $\mathrm{MgO}$ crystal in the natural state, the undeformed $\mathrm{MgO}$ crystal is fixed on the horizontal stage of the scanning acoustic microscope. The couplant between the lens and the sample surface is water with the temperature of $60^{\circ} \mathrm{C}$, and then the acoustic wave velocity of the couplant in Eq. (58) is $v_{\mathrm{c}}=1551.0 \mathrm{~m} / \mathrm{s}$. The $\mathrm{MgO}$ crystal together with the horizontal stage is rotated by the mechanical system to specify the wave propagating direction. The angle between the propagating direction and the [100] axis is defined as the phase angle. In the experiment, the phase angles are $80^{\circ}, 90^{\circ}$, and ranged from $0^{\circ}$ to $30^{\circ}$ with intervals of $5^{\circ}$. The $V(z)$ curve is measured for every specified phase angle, and the AR model is utilized to determine the period of the dips in the $V(z)$ curve. On knowing the period $\Delta z$, operating frequency $f$, and the couplant velocity $v_{\mathrm{c}}$, Eq. (58) can be used to determine the phase velocities of surface waves propagating along the specified directions.

The experimental results as well as the calculated values are shown in the 'Natural state' columns of Table 1. Figure 9 is the distribution of surface wave velocities of $\mathrm{MgO}$ half-space with $(001)$ orientation, where the experimental data are plotted as solid circles, while the thicker and thinner lines are the calculated surface wave velocity and the limiting velocity, respectively.

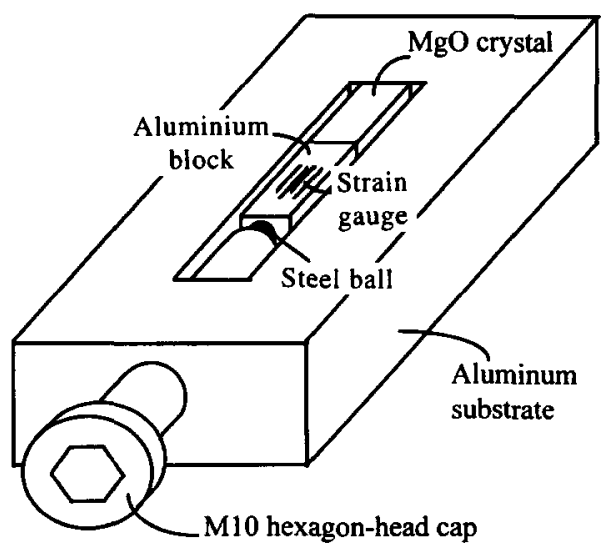

Figure 8 Loading system to create uniaxial compression in the MgO crystal 


\begin{tabular}{|c|c|c|c|c|}
\hline \multirow{3}{*}{$\begin{array}{l}\text { Phase angle } \\
\text { (deg.) }\end{array}$} & \multicolumn{4}{|c|}{ Surface wave velocity $v_{R}(\mathrm{~m} / \mathrm{s})$} \\
\hline & \multicolumn{2}{|c|}{ Experimental data } & \multicolumn{2}{|c|}{ Calculated values } \\
\hline & Natural state & Initial state & Natural state & Initial state \\
\hline 0.0 & 5518.54 & 5522.68 & 5522.05 & 5523.03 \\
\hline 5.0 & 5522.50 & - & 5526.33 & \\
\hline 10.0 & 5537.20 & 5539.30 & 5538.67 & 5539.59 \\
\hline 15.0 & 5563.55 & - & 5558.13 & - \\
\hline 20.0 & 5586.10 & 5588.91 & 5582.41 & 5583.19 \\
\hline 25.0 & 5606.47 & - & 5606.16 & - \\
\hline 30.0 & 5620.13 & - & 5574.19 & - \\
\hline 80.0 & 5539.91 & 5538.56 & 5538.67 & 5537.76 \\
\hline 90.0 & 5520.90 & 5519.12 & 5522.05 & 5521.13 \\
\hline
\end{tabular}

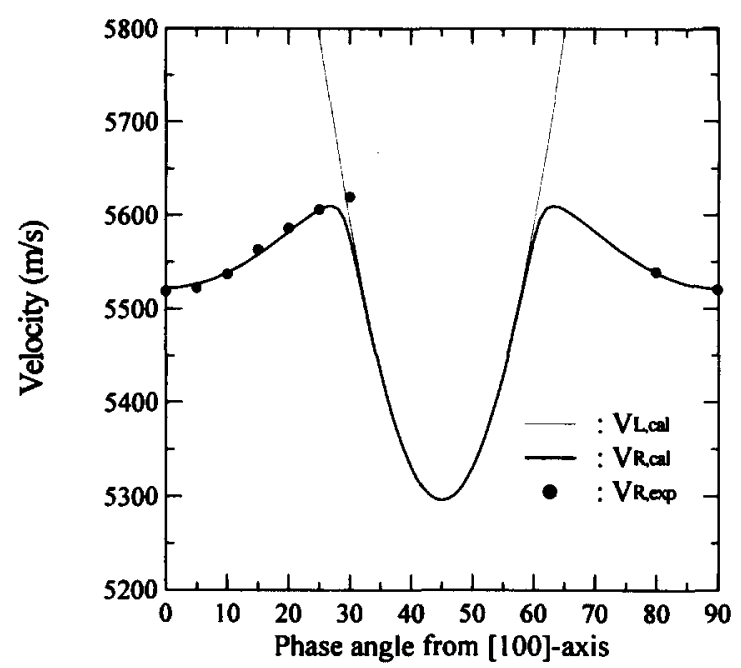

Figure 9 Surface velocity of $\mathrm{MgO}$ crystal with (001) orientation

We note, the measured velocity at the phase angle of $30^{\circ}$ is much larger than the calculated surface wave velocity, because that for particles on the (001) surface of a cubic half-space, the polarization due to surface waves is dominant by the shear horizontal ( $\mathrm{SH})$ component as the phase angles are within the range of about $30^{\circ}$ to $60^{\circ}$ relative to the [100] axis. Therefore, no leaky signal due to the surface waves with the SH component can be received by the lens. In fact, the velocity determined from the measured $V(z)$ curve at $30^{\circ}$ is the velocity of the pseudo surface wave ${ }^{[31]}$. Because the dominant components of the corresponding surface polarization are parallel to the propagating direction and perpendicular to the (001) surface, the leaky signal due to the pseudo surface wave can easily propagate through the water and be detected by the lens.

Fastening the screw loading device, a uniaxial compressive strain is read from the strain indicator as $\epsilon_{11}=-392 \times 10^{-6}$, and the corresponding initial stress $\tau_{I J}^{\mathrm{i}}$ can be determined from Eq. (59). The $V(z)$ curves of the $\mathrm{MgO}$ crystal under this prestressed condition are measured to determine the associated surface wave velocities. The phase angles adopted in this
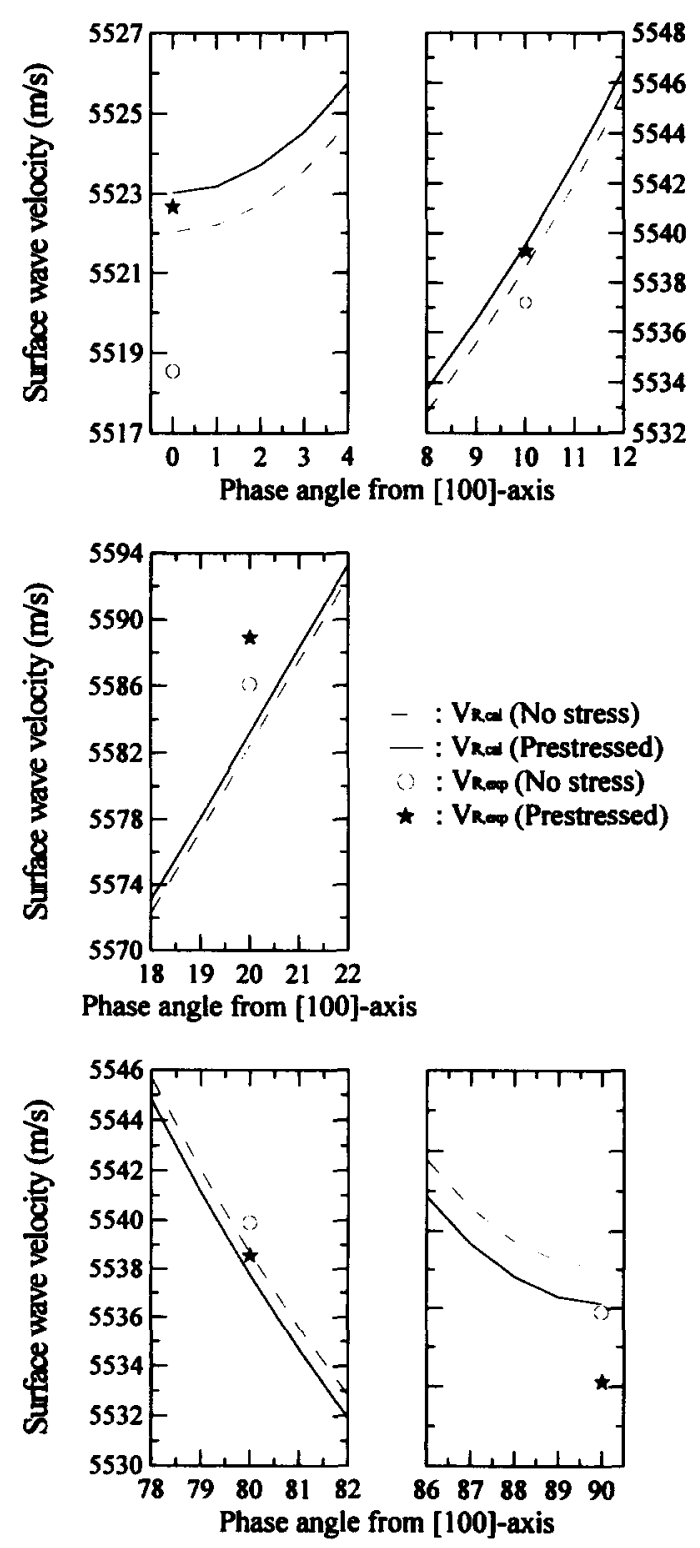

Figure 10 Comparison of surface wave velocities of $\mathrm{MgO}$ crystal in the undeformed natural state and the prestressed initial state 
experiment are $0.0^{\circ}, 10^{\circ}, 20^{\circ}, 80^{\circ}$, and $90^{\circ}$. The experimental results are listed in the 'Initial state' column of Table 1, located under the 'Experimental data' item. The associated phase velocities are also determined by the extended Barnett-Lothe's integral formalism with the procedures shown in Figure 3, and the results are listed in the 'Initial state' column of Table 1, located under the 'Calculated values' item. It is seen from Table 1 that the velocities are increased as the surface waves propagating in phase angles near the [100] axis, parallel to the compression direction; and decreased near the [010] axis, perpendicular to the compression direction. The experimental data and the calculated values for both the nonstressed natural state and the prestressed initial state are plotted in Figure 10. It is seen that the trend of the variation of the surface vave velocities due to the uniaxial compression measured is in agreement with that determined by the numerical calculation.

\section{Conclusion}

In this paper, the surface wave velocities of a prestressed anisotropic crystal are studied. The governing equations and the boundary conditions of the small field superposed on the prestressed crystals are derived by acoustoelasticity and are solved by the extended Barnett-Lothe's integral formalism. The surface wave velocities of the prestressed crystal are dependent on the properties of the crystal, the half-space orientation, the propagating direction as well as the stress and strain in the initial state. From the numerical implementation, the velocities for $\mathrm{Si}$ are increased as the surface waves propagating near the [100] axis, parallel to the tension direction, and decreased near the [010] axis, perpendicular to the tension direction. However, the trend of velocity variation due to the same prestress is opposite to the case of $\mathrm{MgO}$ crystal, i.e., the velocity is decreased near the [100] axis and increased near the [010] axis. In the experiment, a uniaxial compression created by the screw loading system is applied on the MgO crystal along the [100] axis, and the velocities of surface waves propagating on the (001) surface of the prestressed MgO crystal are determined by the period of dips in the $V(z)$ curves of the line focused scanning acoustic microscope. The variation trend of the surface wave velocity due to the initial stress measured in the experiment is in agreement with that determined numerically.

\section{Acknowledgements}

This investigation was supported in part by the National Science Council of the Republic of China through grant NSC 83-0401-E-002-11?.

\section{References}

1 Viktorov, I. A. Rayleigh and Lamb Waves, Plenum press, New York, USA (1967)
2 Farnell, G. W. 'Properties of elastic surface waves' in Physical Acoustics ed. W. P. Mason and R. N. Thurston, Academic Press, New York, Vol 6 (1970) pp 109-166

3 Hirao, M., Sotani, Y., Takami, K. and Fukuoka, H. 'Rayleigh wave propagation in a solid with a cold-worked surface layer' $J$ Nondestr Eval 2 (1981) pp 43-49

4 Synge, J. L. 'Elastic waves in anisotropic media' J. Math. Phys. 35 (1957) pp 323-334

5 Barnett, D. M., Lothe, J., Nishioka, K. and Asaro, R. J. 'Elastic surface waves in anisotropic crystals: a simplified method for calculating Rayleigh velocity using dislocation theory' $J$ Phys $F$ Metal Phys 3 (1973) pp 1083-1096

6 Barnett, D. M., Nishioka, K. and Lothe, J. 'Surface waves in anisotropic media. A formulation for the purpose of rapid numerical calculations' Phys Stat Sol B 55 (1973) pp K115-K117

7 Barnett, D. M. and Lothe, J. 'Considerations of the existence of surface wave solutions in anisotropic elastic crystals' $J$ Phys $F$ Metal Phys 4 (1974) pp 671-686

8 Lothe, J. and Barnett, D. M. 'On the existence of surface wave solutions for anisotropic elastic half-spaces with free surface' $J A p p l$ Phys 47 (1976) pp 428-433

9 Stroh, A. N. 'Steady state problems in anisotropic elasticity' $J$ Math Phys 41 (1962) pp 77-103

10 Wu, T.-T. and Chai, J.-F. 'Propagation of surface waves in anisotropic solids: theoretical calculation and experiment' Ultrasonics 32 1 (1994) pp $21-29$

11 Chai, J.-F. and $\mathbf{W u}$, T.-T. 'Determinations of anisotropic elastic constants using laser generated surface waves' $J$ Acoust Soc Am 956 (1994) pp 3232-3241

12 Eringen, A. C. Mechanics of Continua, Chap. 5. (2nd Ed.), R. E. Krieger Pub., Huntington, N.Y. (1980)

13 Truesdell, C. 'General and exact theory of waves in finite elastic strain' Arch Ratl Mech Anal 8 (1961) pp 263-296

14 Toupin, R. A. and Bernstein, B. 'Sound waves in deformed perfectly elastic materials; the acoustoelastic effect' $J$. Acoust Soc Am 33 (1961) pp 216-225

15 Pao, Y. H., Sachse, W. and Fukuoka, H. 'Acoustoelasticity and ultrasonic measurements of residual stresses' in Physical Acoustics ed. W. P. Mason and R. N. Thurston, Academic Press, New York, Vol 17 (1984) pp 62-143

16 Thurston, R. N. 'Effective elastic coefficients for wave pro-pagation in crystals under stress' $J$ Acoust Soc Am 372 (1965) pp 348-356

17 Thurston, R. N. 'Wave propagation in fluids and normal solids' in Physical Acoustics ed. W. P. Mason, Academic Press, New York, Vol 1, Part A (1964) pp 1-110

18 Hayes, M. and Rivlin, R. S. 'Surface waves in deformed elastic materials' Arch Ratl Mech Anal 8 (1961) pp 358-380

19 Iwashimizu, Y. and Kobori, $\mathbf{O}$. 'The Rayleigh wave in a finitely deformed isotropic elastic material' $J$ Acoust Soc Am 643 (1978) pp 910-916

20 Chadwick, P. and Jarvis, D. A. 'Surface waves in a prestressed elastic body' Proc Roy Soc Lond A.366 (1979) pp 517-536

21 Mase, G. T. and Johnson, G. C. 'An acoustoelastic theory for surface waves in anisotropic media' $J$ Appl Mech 54 (1987) pp 127-135

22 Lemons, R. A. and Quate, C. F. 'Acoustic microscopy' in Physical Acoustics ed. W. P. Mason and R. N. Thurston, Academic Press, New York, Vol 14 (1979) pp 1-92

23 Quate, C. F., Atalar, A. and Wickramasinghe, H. K. 'Acoustic microscopy with mechanical scanning - a review' Proc IEEE 67 (1979) pp 1092-1113

24 Weglein, R. D. 'A model for predicting acoustic material signatures' Appl Phys Lett 34 (1979) pp 179-181

25 Bertoni, H. L. 'Ray-optical evaluation of $V(z)$ in the reflection acoustic microscope' IEEE Trans Sonics Ultras SU-31 2 (1984) pp 105-116

26 Kushibiki, J. I. and Chubachi, N. "Material characterization by linefocus-beam acoustic microscope' IEEE Trans Sonics Ultra SU-32 2 (1985) pp 189-212

27 Kim, J. O. and Achenbach, J. D. 'Elastic constants of single-crystal transition-metal nitride films measured by line-focus acoustic microscopy' J Appl Phys 725 (1992) pp 1805-1811

28 Lothe, J. and Barnett, D.M. 'Integral formalism for surface waves in piezoelectric crystals. Existence considerations' $J$ Appl Phys 475 (1976) pp 1799-1807

29 Chai, J.-F. and $\mathbf{W u}, \mathbf{T}$.-T. 'Propagation of surface waves in a prestressed piezoelectric material' $J$ Acoust Soc Am to be published

30 System Identification Toolbox User's Guide The Math Works, Inc. MA, USA (1991) 
31 Lim, T. C. and Farnell, G. W. 'Character of pseudo surface waves on anisotropic crystals' J Acoust Soc Am 454 (1969) pp 845-851

32 McSkimin, H. J. and Andreatch JR, P. 'Measurment of third-order moduli of silicon and germanium' J Appl Phys 3511 (1964) pp 3312-3319

33 Bogardus, E. H. 'Third-order elastic constants of $\mathrm{Ge}, \mathrm{MgO}$, and Fused $\mathrm{SiO}_{2}$ ' J Appl Phys 368 (1965) pp 2504-2513

\section{Appendix}

\section{Material constants of cubic crystals}

(I) Density and the second order elastic constants

\begin{tabular}{lllll}
\hline & & \multicolumn{3}{c}{ Elastic constants $(\mathrm{GPa})$} \\
\cline { 4 - 5 } Crystal & $\begin{array}{l}\text { Density } \rho \\
\left(\frac{\mathrm{kg}}{\mathrm{m}^{3}}\right)\end{array}$ & $C_{11}$ & $C_{12}$ & \multicolumn{1}{c}{$C_{44}$} \\
\hline $\mathrm{Si}^{[32]}$ & 2332.0 & 165.7 & 63.9 & 79.56 \\
$\mathrm{Mg0}^{[33]}$ & 3598.0 & 301.02 & 99.19 & 157.65 \\
\hline
\end{tabular}

(II) The third order elastic constants

\section{Elastic constants $(\mathrm{GPa})$}

\begin{tabular}{llllrrr}
\cline { 2 - 6 } Crystal & $C_{111}$ & $C_{112}$ & $C_{123}$ & $C_{144}$ & $C_{166}$ & $C_{456}$ \\
\hline $\mathrm{Si}^{[32]}$ & -825.0 & -451.0 & -64.0 & 12.0 & -310.0 & -64.0 \\
$\mathrm{MgO}^{[33]}$ & -4895.0 & -95.0 & -69.0 & 113.0 & -659.0 & 147.0 \\
\hline
\end{tabular}

